\title{
Transformation of a cold to hot tumor and a durable response to immunotherapy in a patient with non-small cell lung cancer after chemoradiotherapy: a case report
}

\author{
Qian Miao, Longfeng Zhang, Xiaobin Zheng, Kan Jiang, Biao Wu, Gen Lin \\ Department of Thoracic Oncology, Fujian Cancer Hospital and Fujian Medical University Cancer Hospital, Fuzhou, China \\ Correspondence to: Gen Lin, MD, PhD. Department of Thoracic Oncology, Fujian Cancer Hospital and Fujian Medical University Cancer Hospital, \\ Fuma Road 420, Fuzhou 350014, China. Email: lingen197505@163.com.
}

\begin{abstract}
Immune checkpoint inhibitors (ICIs) have become an important milestone in the treatment of non-small cell lung cancer (NSCLC). High expression of protein ligand 1 (PD-L1) and tumor mutation burden (TMB) can help to select the dominant population for immunotherapy, but the expression of PDL1 does not seem to be unchanged. A 61-year-old man with adenocarcinoma of the lung experienced postoperative recurrence. PD-L1 expression was negative before recurrence, and TMB was stable by nextgeneration sequencing (NGS) test. However, after radiotherapy and chemotherapy, PD-L1 positive expression was found in a re-biopsy specimen, and NGS detection indicated the loss of immune negative predictive genes. The patient achieved a durable response to a posterior-line immunotherapy combined chemotherapy. The tumor microenvironment maybe changed after chemoradiotherapy, which provides an opportunity for patients to benefit from immunotherapy. The use of NGS in dynamic detection and PD-L1 expression may help monitor this change in the tumor microenvironment, the transition from cold to hot tumor. This case maybe provides new clinical evidence that a non-immuno-dominant population in the initial state can be converted to a population with the benefit of immunotherapy after chemoradiotherapy. However, patients who are initially unsuitable for immunotherapy may still need to undergo combined immunotherapy to achieve a clinical benefit.
\end{abstract}

Keywords: Non-small cell lung cancer (NSCLC); chemoradiotherapy; immunotherapy; next-generation sequencing (NGS); protein-ligand 1 (PD-L1); case report.

Submitted Feb 24, 2021. Accepted for publication Apr 21, 2021.

doi: 10.21037/apm-21-761

View this article at: http://dx.doi.org/10.21037/apm-21-761

\section{Introduction}

Therapies with inhibitors of programmed death-1 (PD-1) or protein-ligand 1 (PD-L1) are effective and may achieve long-term control for patients with advanced non-small cell lung cancer (NSCLC). Monotherapies of immune checkpoint inhibitors (ICIs) have shown rapid and durable responses in $14-20 \%$ of pre-treated patients with advanced NSCLC. The response rate is about $45 \%$ in the untreated population with high PD-L1 expression, and can reach about $50-60 \%$ when combined with chemotherapy and/or anti-vascular treatment.
Recent epidemics have unofficially divided tumors into "hot" and "cold" categories, of which the former is more responsive to immunotherapy than the latter (1). In addition to the presence of tumor-infiltrating lymphocytes (TILs), expressions of PD-L1 and tumor-related immune cells, possible genomic instability, and the presence of a pre-existing antitumoral immune response have all been described as characteristics of hot tumors. Cold tumors have been described as having the converse symptoms of hot tumors (2). Recently, a novel theory proved the evolution of cancer in the metastatic stage, highlighted the development model of tumors, and proposed a mechanism 
for the transformation of tumors from cold to hot (3). In the future, single-agent immunotherapy may be difficult to meet the needs of most cancer patients, and immunotherapy combined with chemotherapy, anti-vascular drugs or other target immunosuppressants may enhance weak antitumor immunity and increase the effects of immunotherapy.

In this paper, we discuss a patient with NSCLC, who relapsed after surgery and received combined immunotherapy. PD-L1 detection and next-generation sequencing (NGS) for gene detection were performed on postoperative specimens, and specimens that relapsed after the patient received radiotherapy and chemotherapy. The effects of radiotherapy and chemotherapy on the immune microenvironment were also compared and analyzed. We present the following article in accordance with the CARE reporting checklist. Available at http://dx.doi.org/10.21037/ apm-21-761

\section{Case presentation}

In October 2018, a 61-year-old man with a history of heavy smoker was admitted to our hospital, complaining of a cough and shortness of breath after activity. Diagnostic imaging with chest computed tomography (CT) revealed a peripheral pulmonary mass in the left upper lobe; however, the bilateral hilar and mediastinal lymph nodes were not enlarged. Positron emission tomography/CT (PET/CT) scans confirmed that there were no other metastatic hypermetabolic lesions other than those in the left pulmonary mass. Brain magnetic resonance imaging (MRI) was negative for metastasis. A left upper lobectomy and systematic lymph node dissection were the performed, and a conclusion of primary adenocarcinomas (pT4N0M0, stage IIIA) was reached. The genetic characteristics of the surgical specimen were tested using NGS (FoundationOne CDx, Roche, Switzerland) (4), which showed the presence of ataxia telangiectasia mutated (ATM) mutation, polybromo 1 (PBRM1) mutation, serine/threonine kinase 11 (STK11) mutation, Cyclin D1 (CCND1) amplification, fibroblast growth factor (FGF)3/4/19 amplification. PD-L1 expression was negative in the Dako 28-8.

After 3 cycles of adjuvant chemotherapy of pemetrexed combined with carboplatin, a recurrence was found in the patient's left pleura. The patient subsequently received 60 Gy/30 F chest radiotherapy. However, 12 months later, he experienced multiple recurrences in his left lung. The patient then underwent a re-biopsy of the left lung mass through a CT-guided percutaneous puncture, and the specimen was again submitted for NGS and PD-L1 testing. No amplification of CCND1 and FGF3/4/19 was detected in the samples after chemoradiotherapy, and the expression of PD-L1 changed from negative to positive (see Figure 1). The tumor mutation burden (TMB) was still stable, STK11 and ATM and PBRM1 mutations remained unchanged from the baseline status.

From May 2020, the patient has received 4 courses of atezolizumab plus bevacizumab plus carboplatin plus paclitaxel (ABCP). After 2 months of treatment a partial response was obtained based on the PET/CT assessment. Subsequently, the patient underwent a maintenance treatment of atezolizumab plus bevacizumab every 3 weeks. Efficacy and adverse events were evaluated every 6 weeks. As of the last follow-up date of December 30, 2020, no adverse effects had been found, and a durable response had been made. All procedures performed in studies involving human participants were in accordance with the ethical standards of the institutional and/or national research committee(s) and with the Helsinki Declaration (as revised in 2013). Written informed consent was obtained from the patient for publication of this study and any accompanying images.

\section{Discussion}

Tissue based PD-L1 expression is the first criterion for the prediction of ICI treatment in NSCLC patients. The TMB-H ultimately became the second biomarker approved for immunotherapy. Dako 22C3 pharmDx and FoundationOne CDx are companion diagnostic tests that were approved at the same time (5).

In addition to the above-mentioned biomarkers, some cancer cell-specific carcinogenic genetic events may also predict immunotherapy responses. For example, epidermal growth factor receptor (EGFR), v-raf murine sarcoma viral oncogene homolog B1 (BRAF) V600E Mutations, anaplastic lymphoma kinase (ALK), one ROS proto-oncogene 1 (ROS1), or rearranged during transfection (RET) rearrangements, and mesenchymal-epithelial transition factor (MET) exon 14 mutations generally respond poorly to ICIs (6). STK11 mutation is associated with decreased efficacy in relation to ICIs $(7,8)$. Multiple studies have shown the potential value of CCND1 amplification as a biomarker for predicting negative treatment effects $(9,10)$. Studies by Singavi and others have suggested that the amplification of some genes on the human chromosome 11q13, such as CCND1, FGF3, FGF4, and FGF19, may 


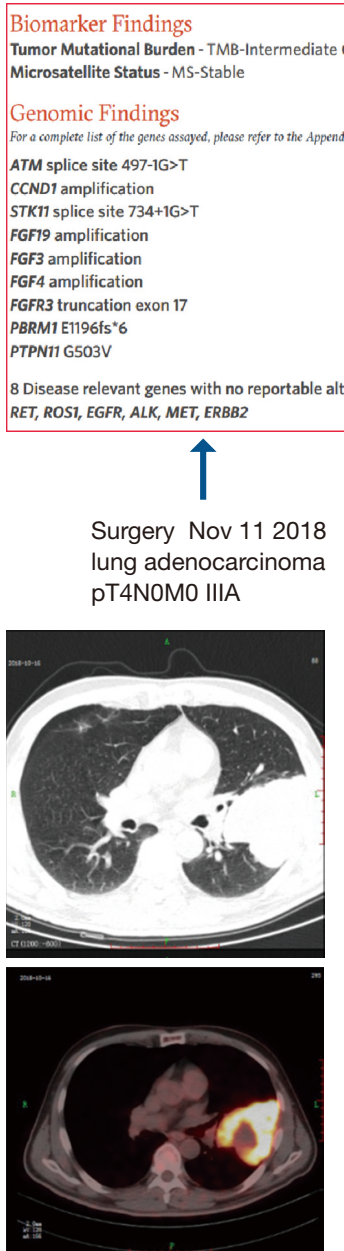

Oct 162018

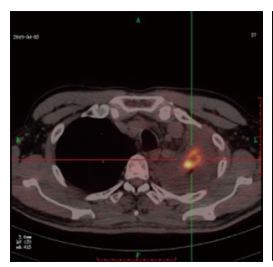

Apr 42019 relapse

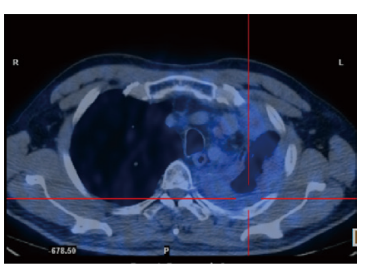

Jul 312019
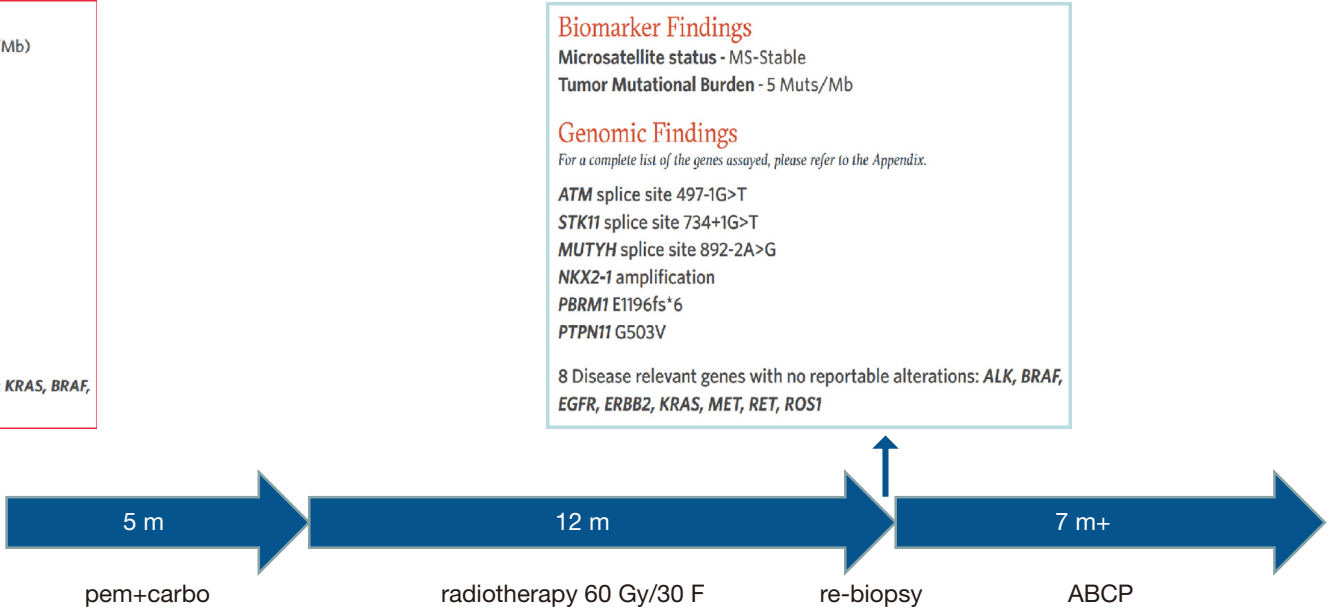

Figure 1 Anti-cancer treatment sequence and genetic profile for the entire timeline and imaging evaluation of the corresponding treatment. ABCP, atezolizumab plus bevacizumab plus carboplatin plus paclitaxel.

be related to the occurrence of hyper-progression (11). The loss of function of the ATM is related to the autosomal recessive disease ataxia-telangiectasia, hypersensitivity to ionizing radiation, cancer susceptibility, immunodeficiency, and genomic instability, which may indicate better immune efficacy and sensitivity to ionizing radiation $(12,13)$. The loss of PBRM1 may change the overall tumor cell expression profile. Its significance in lung cancer is not clear; however, studies have shown that the loss of PBRM1 is related to the clinical benefit of clear cell renal cell carcinoma immunotherapy (14).

The patient had a genotypic profile similar to that of a cold tumor, and was not sensitive to postoperative chemotherapy. After 1 year of radiation control, the CCND1 and FGF genes were lost, and PD-L1 expression changed from negative to positive in the second biopsy specimens after tumor recurrence. It appeared to have transformed from a cold to a hot tumor, and while the STK11 gene was still present, there appeared to be a more favorable prognosis for immunotherapy. Additionally, the long-term effectiveness of the subsequently administered Impower150 mode of immunotherapy was also confirmed. As radiotherapy can currently be delivered with great precision, and induced immunogenic cell death pathways can potentially convert tumors into in-situ vaccines, the efficacy of subsequent immunotherapy can be enhanced $(15,16)$. Chemotherapy can enhance the immunogenicity of tumor cells through antigenic and adjuvant pathways $(17,18)$. Preclinical evidence has suggested that chemotherapy and radiotherapy may up-regulate PD-L1 expression in 
tumor cells $(19,20)$. The parallel occurrence of multiple tumor-promoting mechanisms may eventually lead to the formation of cold tumors. The tumor microenvironment is very complex and will change over the course of treatment. Thus, a combined approach to immunotherapy may still be needed to achieve clinical benefits for patients whose tumors may transform from cold to hot. However, hot or cold tumor is only an artificial parameter division, and these immune contexture parameters are associated with longterm survival and prediction of response to treatments, further analysis of large samples is needed to determine which patients and what methods can be used to transform "cold tumors" into "hot tumors" so that more people can benefit from immunotherapy.

This study had a number of limitations. As a case report, only one patient was examined. Further, the investigation did not consider the mechanism of in vitro transformation of tumors from cold to hot. Finally, the presence of TILs was not determined in this case.

\section{Conclusions}

In conclusion, this case report provides new clinical evidence that a non-immuno-dominant population in the initial state can be converted to a population with the benefit of immunotherapy after chemoradiotherapy.

\section{Acknowledgments}

Funding: None.

\section{Footnote}

Reporting Checklist: The authors have completed the CARE reporting checklist. Available at http://dx.doi.org/10.21037/ apm-21-761

Conflicts of Interest: All authors have completed the ICMJE uniform disclosure form (available at http://dx.doi. org/10.21037/apm-21-761). The authors have no conflicts of interest to declare.

Ethical Statement: The authors are accountable for all aspects of the work in ensuring that questions related to the accuracy or integrity of any part of the work are appropriately investigated and resolved. All procedures performed in studies involving human participants were in accordance with the ethical standards of the institutional and/or national research committee(s) and with the Helsinki Declaration (as revised in 2013). Written informed consent was obtained from the patient for publication of this study and any accompanying images.

Open Access Statement: This is an Open Access article distributed in accordance with the Creative Commons Attribution-NonCommercial-NoDerivs 4.0 International License (CC BY-NC-ND 4.0), which permits the noncommercial replication and distribution of the article with the strict proviso that no changes or edits are made and the original work is properly cited (including links to both the formal publication through the relevant DOI and the license). See: https://creativecommons.org/licenses/by-nc-nd/4.0/.

\section{References}

1. Galon J, Bruni D. Approaches to treat immune hot, altered and cold tumours with combination immunotherapies. Nat Rev Drug Discov 2019;18:197-218.

2. Hegde PS, Karanikas V, Evers S. The Where, the When, and the How of Immune Monitoring for Cancer Immunotherapies in the Era of Checkpoint Inhibition. Clin Cancer Res 2016;22:1865-74.

3. A Angelova M, Mlecnik B, Vasaturo A, et al. Evolution of Metastases in Space and Time under Immune Selection. Cell 2018;175:751-65.e16.

4. Frampton GM, Fichtenholtz A, Otto GA, et al. Development and validation of a clinical cancer genomic profiling test based on massively parallel DNA sequencing. Nat Biotechnol 2013;31:1023-31.

5. Filipovic A, Miller G, Bolen J. Progress Toward Identifying Exact Proxies for Predicting Response to Immunotherapies. Front Cell Dev Biol 2020;8:155.

6. Mhanna L, Guibert N, Milia J, et al. When to Consider Immune Checkpoint Inhibitors in Oncogene-Driven Non-Small Cell Lung Cancer? Curr Treat Options Oncol 2019;20:60.

7. Koyama S, Akbay EA, Li YY, et al. STK11/LKB1 Deficiency Promotes Neutrophil Recruitment and Proinflammatory Cytokine Production to Suppress T-cell Activity in the Lung Tumor Microenvironment. Cancer Res 2016;76:999-1008.

8. Skoulidis F, Goldberg ME, Greenawalt DM, et al. STK11/LKB1 Mutations and PD-1 Inhibitor Resistance in KRAS-Mutant Lung Adenocarcinoma. Cancer Discov 2018;8:822-35.

9. Chen Y, Huang Y, Gao X, et al. CCND1 Amplification 
Contributes to Immunosuppression and Is Associated With a Poor Prognosis to Immune Checkpoint Inhibitors in Solid Tumors. Front Immunol 2020;11:1620.

10. Yu J, Yan J, Guo Q, et al. Genetic Aberrations in the CDK4 Pathway Are Associated with Innate Resistance to PD-1 Blockade in Chinese Patients with Non-Cutaneous Melanoma. Clin Cancer Res 2019;25:6511-23.

11. Singavi AK, Menon S, Kilari D, et al. 1140PDPredictive biomarkers for hyper-progression (HP) in response to immune checkpoint inhibitors (ICI)-analysis of somatic alterations (SAs). Ann Oncol 2017. doi: 10.1093/annonc/ $\operatorname{mdx} 376.006$

12. Hu M, Zhou M, Bao X, et al. ATM inhibition enhances cancer immunotherapy by promoting mtDNA leakage and cGAS/STING activation. J Clin Invest 2021;131:e139333.

13. Kakoti S, Sato H, Laskar S, et al. DNA Repair and Signaling in Immune-Related Cancer Therapy. Front Mol Biosci 2020;7:205.

14. Miao D, Margolis CA, Gao W, et al. Genomic correlates of response to immune checkpoint therapies in clear cell renal cell carcinoma. Science 2018;359:801-6.

Cite this article as: Miao Q, Zhang L, Zheng X, Jiang K, Wu B, Lin G. Transformation of a cold to hot tumor and a durable response to immunotherapy in a patient with non-small cell lung cancer after chemoradiotherapy: a case report. Ann Palliat Med 2021;10(4):4982-4986. doi: 10.21037/apm-21-761
15. Demaria S, Coleman CN, Formenti SC. Radiotherapy: Changing the Game in Immunotherapy. Trends Cancer 2016;2:286-94.

16. Whiteside TL, Demaria S, Rodriguez-Ruiz ME, et al. Emerging Opportunities and Challenges in Cancer Immunotherapy. Clin Cancer Res 2016;22:1845-55.

17. Beatty GL, Gladney WL. Immune escape mechanisms as a guide for cancer immunotherapy. Clin Cancer Res 2015;21:687-92.

18. Zitvogel L, Kepp O, Kroemer G. Decoding cell death signals in inflammation and immunity. Cell 2010;140:798-804.

19. Zhang P, Su DM, Liang M, Fu J. Chemopreventive agents induce programmed death-1-ligand 1 (PD-L1) surface expression in breast cancer cells and promote PD-L1mediated T cell apoptosis. Mol Immunol 2008;45:1470-6.

20. Deng L, Liang H, Burnette B, et al. Irradiation and antiPD-L1 treatment synergistically promote antitumor immunity in mice. J Clin Invest 2014;124:687-95.

(English Language Editor: L. Huleatt) 